\title{
Effect of the Combination of Femtosecond Laser Pulses Exposure on the Etching Rate of Fused Silica in Hydrofluoric Acid
}

\author{
Alexandros Mouskeftaras and Yves Bellouard \\ Galatea Lab, IMT/STI, Ecole Polytechnique Fédérale de Lausanne (EPFL), Rue de la Maladière \\ 71b, CH-2002 Neuchâtel, Switzerland \\ E-mail: alexandros.mouskeftaras@doc.polytechnique.org
}

\begin{abstract}
The paper presents an experimental study of the laser-assisted wet etching of fused Silica. We investigate the mechanisms behind the increased etching rate of laser-exposed regions and use a combined laser pulse as a mean for obtaining better etching rate contrast between pristine and lasermodified silica. This combined pulse consists of two orthogonally polarized pulses with a fixed delay between them. For this purpose, we use a $150 \mathrm{fs}$ laser operating at $1030 \mathrm{~nm}$ and $100 \mathrm{kHz}$ repetition rate integrated in a simple experimental setup where the beam is further divided in two paths, onto which independent laser parameters can be applied. Our results emphasize the role of matrix defects, causing fluorescent emission, in the accelerated etching rate of laser-modified material.
\end{abstract}

DOI: 10.2961/jlmn.2018.01.0006

Keywords: femtosecond laser, fused silica, laser-assisted wet etching, Raman spectroscopy, laserinduced defects

\section{Introduction}

During the past decades pulsed-laser processing has established itself as one of the most flexible micro manufacturing methods. The reason for this is that it offers unique processing quality in almost any material, from metals to polymers but also in glass and ceramics. Even more impressive is the fact that in transparent materials laser processing reaches full, three-dimensional capabilities. This enables their use in many applications such as in photonics in general (see for instance: [1-4]), optical memories [5], micro-fluidics [6,7] but also in micro-mechanics [8,9]. To support this technological development, there is a need to understand the mechanisms involved in the laser processing of silica, and more specifically, the effect on etching rate that we will emphasize later.

Processing of transparent materials can be made by laser at several regimes such as that of ablation and fusion. However, at laser exposures needed to reach these regimes, the presence of a certain number of detrimental effects (large kerf, ablated material deposition, stress fields through shockwave generation, large heat affected zone etc.) limits the machining quality. An alternative is the technique of the laser-assisted wet etching [10] that uses laser exposure to first modify the transparent material and then acid attack (HF) to remove the modified zones at an accelerated rate. This method has been later on extended to fused silica [11] and further extended to arbitrary shapes [12]. However, etching rates, even at the laser affected zones can be quite slow which translates as a moderate etching rate contrast between laser-affected and pristine silica. This has as a result the increase of the taper angle [13-15] of the machined areas and in many cases this it to be avoided.
In this paper, we are exploring the mechanisms behind the HF etching of laser-exposed fused silica with the aim of investigating the possibility for increased etching rate contrast between pristine and modified material.

In the past, several works have demonstrated the benefits from using ultrafast laser bursts such as crack-free processing [16] and limited heat effects [17]. Today, there are commercial tools making it possible to temporally divide a laser pulse into a burst of smaller pulses with variable delay between them (Spatial Light Modulators, Acousto-Optic Modulators, Deformable Mirrors). Recently, several works have made use of this feature to outline the benefits of using pulse trains including optimization of self-arranged nanogratings in bulk fused silica [18], correction of wavefront distortions [19], sub-diffraction limited processing [20] and etching rate enhancement on surface written pits [21]. The motivation for using such pulse envelopes relies on the fact that the initial steps of laser-induced excitation are decoupled and thus, one can obtain optimal control of the energy deposition [22,23]. It is in the aim of this paper to provide further insights in the laser processing of transparent materials with a combination of pulses by focusing on in-volume applications and more in particular fabrication of laser-written microchannels and study of laserwritten tracks.

\section{Experimental Set-Up}

The combined-pulse experimental scheme considered in this work is illustrated in Fig.1. An Yb-based regenerative amplifier (from Amplitude Systèmes), delivering 150 fs pulses (FWHM FROG measurement) at a wavelength of $1030 \mathrm{~nm}$ and pulse repetition frequency of $100 \mathrm{kHz}$ has been used as the laser source. Then, the beam is split in two parts of controllable power by use of a half-wave plate and 
a polarizing, beam-splitting cube. The reflected part (spolarized) can be arbitrarily delayed in time using a manual delay line (two fixed mirrors on a linear translation stage with $0.5 \mu \mathrm{m}$ fine graduation adjustment). Two more halfwave plates on each of the beam paths ensure precise power setting after the second polarizing, beam-splitting cube that is used for recombining the beams. The two beams are tightly focused with a 0.42 numerical aperture (NA) objective (Thorlabs, LMH-20X-1064) producing a focal spot diameter of $\sim 1.5 \mu \mathrm{m}$ measured in air with magnified beam profiler (see microscope on Fig.1). The focal spot may be arbitrarily positioned along the optical axis as the microscope objective is mounted on a motorized, lineartranslation stage (PI-MICOS). The fused silica sample (high $\mathrm{OH}$ content,>1000 ppm) lies on a XY motorized, linear-translation stage (PI-MICOS). The speed of the stages was fixed at $1 \mathrm{~mm} / \mathrm{s}$. In situ transmission microscopy with visible light (Thorlabs, M625L3) was used to observe the modified regions inside the sample. A CMOS detector (IDS, USB 2 uEye SE) recorded the images yielding $\sim 1$ $\mu \mathrm{m}$ spatial resolution. Two different magnifications (20X and 50X) were available by using two microscope objectives (Olympus, MPLFLN20XBDP and MPLFLN50XBDP). Precise spatial overlap of the two pulses was achieved by alignment of two mirrors on Gimbal mounts (Thorlabs, GM100) in a similar way as in a galvanometer scanner. The criterion used for positioning of the beams was that the laser-induced modification was only achieved when the two beams were spatially overlapping. Temporal "zero" delay, corresponding to coincidence of the two pulses intensity maxima, was measured by maximizing the sum-frequency generation signal on a photodetector. For that a BBO crystal was used along with a prism to separate the fundamental from the second harmonic (not shown here). The temporal resolution was estimated to be of the order of the pulse duration ( 150 fs).

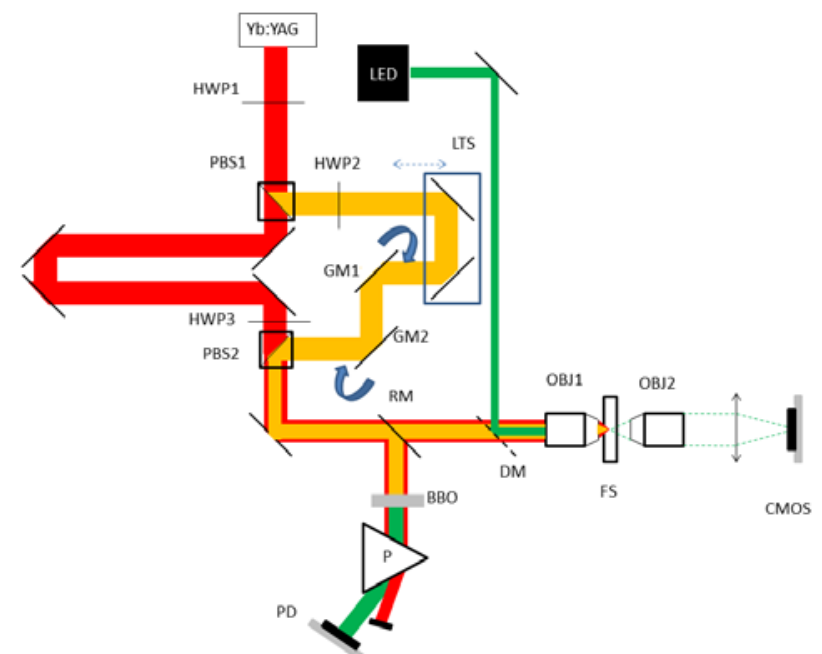

Fig. 1 Experimental setup for combined-pulse laser processing. HWP: Half Wave Plate, PBS: Polarizing Beam-Splitting Cube, LTS: Linear Translation Stage, GM: Gimbal Mount, LED: Light Emitting Diode, RM: Removable Mirror, DM: Dichroic Mirror, P: Prism, BBO: Beta Barium Borate Cristal, PD: Photo Diode, OBJ: Microscope Objective, FS: Fused Silica Substrate, CMOS: Complementary Metal Oxide Semiconductor Detector

\section{Results}

For the experiments performed during this study, the focal spot has been placed at $50 \mu \mathrm{m}$ from the surface, inside a $1 \mathrm{~mm}$ thick fused silica substrate (25 mm square) and lines of constant deposited energy (defined as the amount of energy passing through a given surface element equal to the spot area in the waist area) were written. Total pulse energy varied from $250 \mathrm{~nJ}$ to $2.5 \mu \mathrm{J}$. Lines were always written perpendicularly to the laser beam propagation on unmodified material and separated by $50 \mu \mathrm{m}$ at least from each other. Three different conditions were tested : single pulse with polarization parallel to the writing direction, single pulse with polarization perpendicular to the writing direction and a pulse consisting of the combination of the two previous pulses with 100 fs delay with each other (parallel polarization arriving first). Then, the sample's cross section was polished in order to reveal the laserwritten line and allow penetration of the etchant. Finally, the samples were placed in a $2.5 \%$ hydrofluoric (HF) acid solution for 2 hours. The etched track lengths were measured with an optical microscope. The calculated etching rates are presented on Fig. 2 along with the error bars extracted from repeatability of the measurements over four written lines for each parameter.

A universal increase of the etching rate is observed for increasing pulse energies. Here, it is to be noted that peak power $\mathrm{P}_{\text {peak }}$ for the single pulse case has been kept below 8 MW as to avoid having severe effects from filamentation (when $\mathrm{P}_{\text {peak }}>>\mathrm{P}_{\text {crit }}, \mathrm{P}_{\text {crit }} \sim 3 \mathrm{MW}$ for fused silica [24]). In the combined pulse case the total pulse energy may go well beyond as the electric fields of the two pulses are perpendicular and thus not additive. When delivering the laser energy in a single pulse we observe strong polarization dependence consistent with previous experiments [25]. Under the assumption that we are in the nanograting formation regime, when the electric field is oriented perpendicular to the writing direction the nanogratings orientation is along the laser-written line thus facilitating the advance of the etchant [25]. Most remarkable is the fact the etching rates for the combined pulse energy delivery are constantly above those of the single pulse resulting in a maximum amplification of $\sim 22 \%$ (for $800 \mathrm{~nJ}$ pulse energy). Moreover even at more modest pulse energies we observe that at constant etching rate ( $70 \mu \mathrm{m} /$ hour) we are able to divide the pulse energy by a factor of two (166 nJ for a combined pulse compared to $400 \mathrm{~nJ}$ for a single pulse, perpendicularly polarized). It is to be noted here that $400 \mathrm{~nJ}$ is not the energy threshold for increased etching rate but rather the value at which laser-written tracks started being visible with our imaging setup. The maximum etching rate has been found to be $\sim 122 \mu \mathrm{m} /$ hour measured for a combined pulse of $2.4 \mu \mathrm{J}$. For comparison the etching rate of unmodified fused silica in the same experimental conditions is 3 $\mu \mathrm{m} /$ hour [26] leading to a 40:1 etching contrast. 


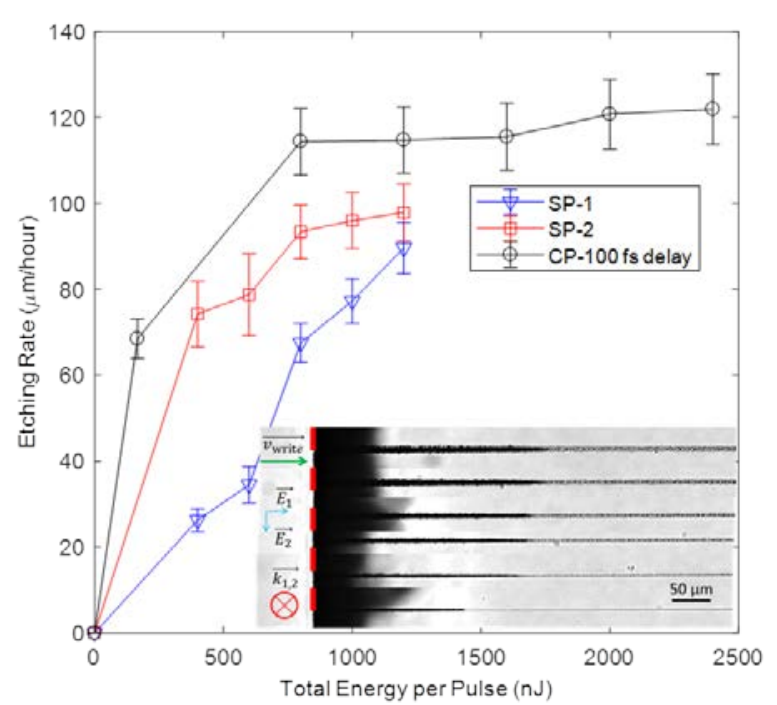

Fig. 2 Measured etching rates in fused silica as a function of the laser pulse energy. SP: Single Pulse, CP: Combined Pulse. Inset figure: optical micrograph of the top view of the fabricated microchannels using a combined pulse. From bottom to top the total energy per pulse is varied from $200 \mathrm{~nJ}$ to $2.4 \mu \mathrm{J}$. Dashed red line indicated the air/glass interface. The different polarization, writing and propagation directions are shown on the inset figure.

In order to further characterize the modifications obtained by combined-pulse processing, we have performed micro-Raman spectroscopic measurements on the cross sections of the induced features. The instrument used was a Lab Ram Aramis confocal Raman microscope by Horiba. The excitation wavelength of the laser used was $523 \mathrm{~nm}$ and averaging over two spectra was always performed. Raman spectroscopy is known to provide information about the molecular structure. Different vibrational bands have been associated with particular ring structures in a random network configuration. More in particular, it is of common knowledge that $440 \mathrm{~cm}^{-1}\left(\omega_{1}\right), 495 \mathrm{~cm}^{-1}\left(D_{1}\right)$, and $606 \mathrm{~cm}^{-1}\left(\mathrm{D}_{2}\right)$, peaks in the Raman spectrum (RS) are signatures of the 6- and 5- fold, 4-fold and 3- fold ring structures respectively. Thus, comparison of these peaks with those of pristine silica can provide information on the laserinduced densification. On figure 3, top plot, we present the measured RS of pristine, laser-affected (by combinedpulse) and laser-affected followed by annealing fused silica. Pulse energy was $1.2 \mu \mathrm{J}$ for the presented case. The raw RS shows an intense fluorescence (FL) signal at long wavenumbers which is associated with laser-induced defects and more precisely non-bridging oxygen hole centers (NBOHC) $[27,28]$. Under these circumstances it is very difficult to perform analysis of the RS as the fluorescence signal has to be removed with an appropriate fit in order to reveal the different Raman peaks of fused silica. Moreover, an increased mathematical treatment of RS adds more free parameters to control that tend to deteriorate the quality of conclusions drawn from these measurements. Therefore, it is common to thermally treat the samples in order to remove the FL signal. The samples containing the laserwritten tracks have been annealed for 10 hours at $300^{\circ} \mathrm{C}$. These conditions were chosen in order to minimize any relaxation of the laser-induced densification while maximizing removal of the FL [29].
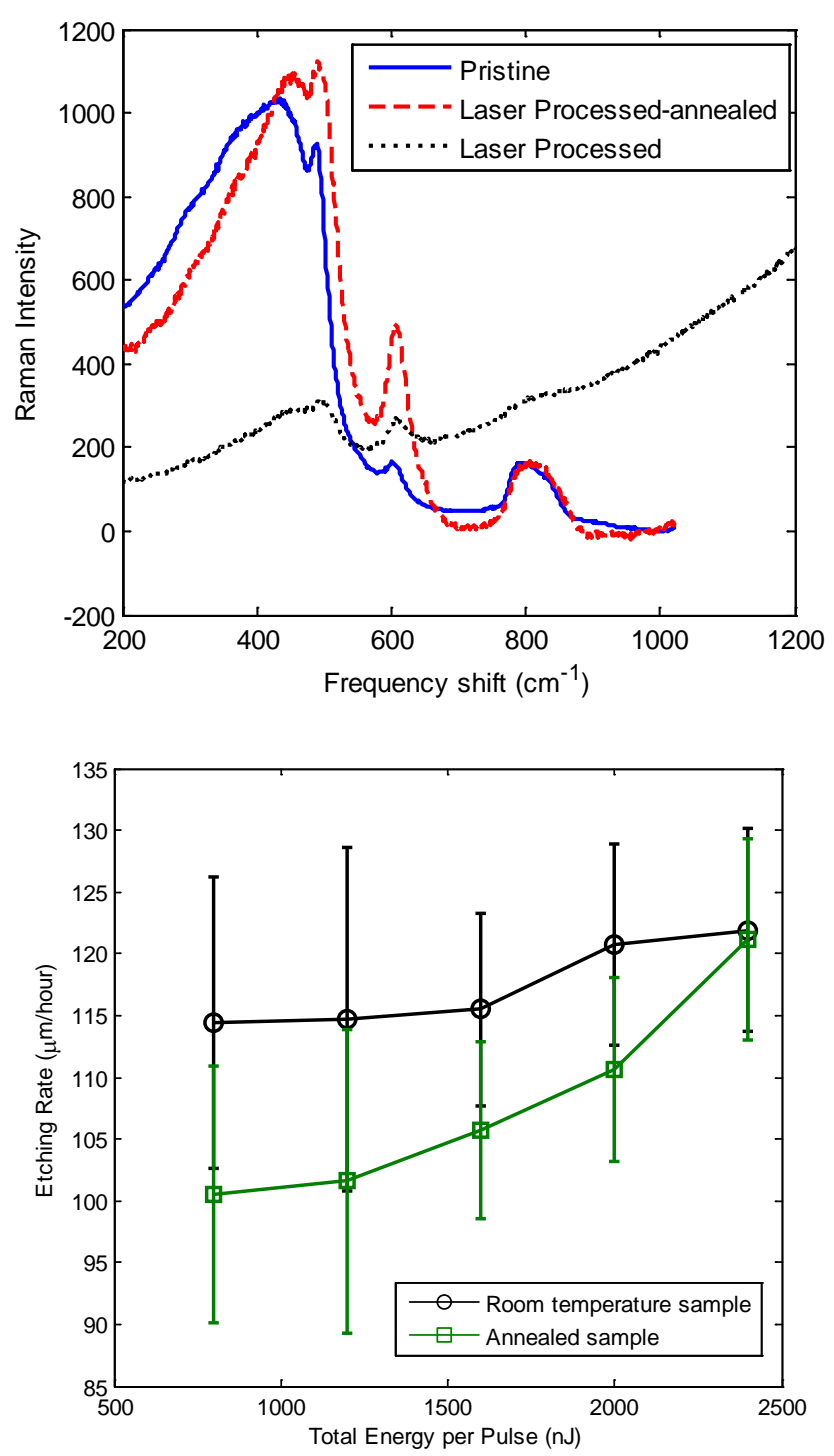

Fig. 3 Top: Raman spectra of in-volume tracks written with a combined-, femtosecond laser pulse. Energy per pulse is $1.2 \mu \mathrm{J}$. Spectrum of pristine silica is presented for comparison. Bottom: Etching rate measurements for laser-written tracks with a combined pulse. Comparison between tracks that were kept at room temperature and tracks that have been annealed at $300^{\circ} \mathrm{C}$ for 10 hours.

As RS is a relatively complex characterization tool where sophisticated analysis must take place in order to give quantitative information, we choose to restrain interpretations at qualitative level by comparing frequency shifts of different peaks associated with n-fold ring structures. Before doing so we subtract a constant baseline from the laser-processed-annealed signal corresponding to residual FL that could not be removed by our annealing treatment. Then we normalize the RS by using the integral of the $\omega_{3}$ peak $\left(800 \mathrm{~cm}^{-1}\right)$ associated with the pure bending (and some stretching of the Si atom). From the treated spectrum (labeled Laser processed-annealed) we can see a shift towards higher wavenumbers for all of the $\omega_{1}, D_{1}$ and $\mathrm{D}_{2}$ peaks. Moreover, a narrowing of the main band is observed (contributions from $\omega_{1}$ and $D_{1}$ ) while the amplitudes of the $\mathrm{D}_{1}$ and $\mathrm{D}_{2}$ peaks become considerably higher. $\mathrm{A}$ measurement of the $D_{1} / D_{2}$ ratio indicates a considerably 
lower value for the laser affected zone ( 2.26) compared to the ratio for pristine silica $(\sim 5.54)$. We can actually measure the difference of the $\mathrm{D}_{2}$ peak position $\left(607 \mathrm{~cm}^{-1}\right)$ from the case of pristine silica $\left(601 \mathrm{~cm}^{-1}\right)$ and thus deduce a shift of approximately $6 \mathrm{~cm}^{-1}$. Comparing with literature data for mechanically densified silica [30] we find a densification of approximately $8 \%$ which is in accordance with similar measurements performed by Bellouard et al. [31].

Finally, we have been interested in measuring the etching rate of the annealed, laser-written features and compare them with the intact ones. These measurements are presented on Figure 3, bottom plot for the combined-pulse case. The etching rate is found to be higher $(15 \%)$ for the sample that stayed at room temperature as compared to the one that has been annealed. As a reminder, the annealed samples have the FL of the laser-induced defects removed while those at room temperature have present a strong FL activity related to them. However, the difference in the etching rate tends to disappear for higher pulse energies. A possible explanation to this is that for moderate pulse energies (below $2 \mu \mathrm{J}$ ) the laser-induced defects have some contribution to the etching rate but in overall the amplified etching rate comes from the fact that the laser has induced modification of the silica. At higher energies, permanent damage of the material accompanied with localized explosion could have taken place thus resulting in annealing independent etching rate.

\section{Conclusion}

In conclusion, we have used laser-assisted wet etching technique to machine channels inside the bulk of fused silica. Our results show that one way of accelerating the etching rate is to use polarization perpendicular to the writing direction of the beam as suggested by literature [25]. More importantly, it has been shown that splitting the pulse energy into two, orthogonally -polarized pulses separated by 100 fs delay significantly improves the etching rate as compared to the case of a single pulse. Furthermore, we have performed Raman Spectroscopy on the laser-written lines and we have found that increased densification is present. Finally, the thermal stability of the etching rates was measured and these results suggest some minor dependence of the etching rate on laser-induced defects. In overall, we show that the use of a combined-pulse provides optimized energy deposition that can be beneficial for laser-induced machining of transparent materials. The reason behind this is going to be the object of further studies as both tailored polarization, longer pulse duration and double-pulse effect may contribute to the improved energy deposition. The simple experimental scheme described in this paper offers the freedom of independently changing energy, pulse duration, delay and wavelength of each of the laser beams

\section{Acknowledgments}

This work is supported by the European Research Council (Grant No. ERC-2012-StG-307442). The authors acknowledge the sponsoring of Richemont International.

\section{References}

[1] K. M. Davis, K. Miura, N. Sugimoto, and K. Hirao: Opt. Lett., 21, (1996) 1729.
[2] Y. Sikorski, A. A. Said, P. Bado, R. Maynard, C. Florea, and K. A. Winick: Electron. Lett., 36, (2000) 226.

[3] K. Minoshima, A. M. Kowalevicz, I. Hartl, E. P. Ippen, and J. G. Fujimoto: Opt. Lett., 26, (2001) 1516.

[4] R. Osellame, S. Taccheo, G. Cerullo, M. Marangoni, D. Polli, R. Ramponi, P. Laporta, and S. De Silvestri : Electron. Lett., 38, (2002) 964.

[5] J. Zhang, M. Gecevičius, M. Beresna, and P. G. Kazansky: Phys. Rev. Lett., 112, (2014) 033901.

[6] K. Sugioka, and Y. Cheng: Lab Chip, 12, (2012) 3576.

[7] Y. Cheng, K. Sugioka, and K. Midorikawa: Opt. Lett., 29, (2004) 2007.

[8] Y. Bellouard, A. A. Said, and P. Bado: Opt. Express, 13, (2005) 6635.

[9] Y. Bellouard: Opt. Mater. Express, 1, (2011) 816.

[10] Y. Kondo, J. Qiu, T. Mitsuyu, K. Hirao, and T. Yoko: Jpn J. Appl. Phys., 38, (1999) L1146.

[11]A. Marcinkevičius, S. Juodkazis, M. Watanabe, M. Miwa, S. Matsuo, H. Misawa, and J. Nishii: Opt. Lett., 26, (2001) 277.

[12] Y. Bellouard, A. Said, M. Dugan, and P. Bado: Opt. Express, 12, (2004) 2120.

[13] S. Kiyama, S. Matsuo, S. Hashimoto, and Y. Morihira: J. Phys. Chem. C, 113, (2009) 11560.

[14]F. He, Y. Cheng, Z. Xu, Y. Liao, J. Xu, H. Sun, C. Wang, Z. Zhou, K. Sugioka, K. Midorikawa and Y. Xu: Opt. Lett., 35, (2010) 282.

[15] K. C. Vishnubhatla, N. Bellini, R. Ramponi, G. Cerullo and R. Osellame: Opt. Express, 17, (2009) 8685

[16] P. R. Herman, A. Oettl, K. P. Chen, and R. S. Marjoribanks: Proc. SPIE, San Jose, (1999) p. 148.

[17] C. Kerse, H. Kalaycığlu, P. Elahi, B. Çetin, D. K. Kesim, Ö. Akçaalan, S. Yavaş, M. D. Aşık, B. Öktem, H. Hoogland, and R. Holzwarth: Nature, 537, (2016) 84.

[18] C. Mauclair, M. Zamfirescu, J. P. Colombier, G. Cheng, K. Mishchik, E. Audouard, and R. Stoian: Opt. Express, 20, (2012) 12997.

[19] A. Mermillod-Blondin, C. Mauclair, A. Rosenfeld, J. Bonse, I. V. Hertel, E. Audouard, and R. Stoian: Appl. Phys. Lett., 93, (2008) 021921.

[20] L. Englert, M. Wollenhaupt, L. Haag, C. SarpeTudoran, B. Rethfeld, and T. Baumert: Appl. Phys. A, (2008) 749.

[21] M. Zhao, J. Hu, L. Jiang, K. Zhang, P. Liu, and Y. Lu: Sci. Rep., 5, (2015) 13202.

[22]A. Mouskeftaras, S. Guizard, N. Fedorov, and S. Klimentov: Appl. Phys. A, 110, (2013) 709.

[23] S. Guizard, S. Klimentov, A. Mouskeftaras, N. Fedorov, G. Geoffroy, and G. Vilmart: Appl. Surf. Sci., 336, (2015) 206.

[24] A.Couairon and A.Mysyrowicz: Phys. Rep., 441, (2007) 47

[25] C. Hnatovsky, R. S. Taylor, E. Simova, V. R. Bhardwaj, D. M. Rayner, and P. B. Corkum: Opt. Lett., 30, (2005) 1867.

[26] Y. Bellouard, A. Said, M. Dugan, and P. Bado: Proc. MRS Online Proceedings Library Archive, (2003), p. 63.

[27] J. W. Chan, T. Huser, S. Risbud, and D. M. Krol: Opt. Lett., 26, (2001) 1726. 
[28] M. Stapelbroek, D. L. Griscom, E. J. Friebele, and G. H. Sigel: J. Non Cryst. Solids, 32, (1979) 313.

[29] J. J. Witcher, W. J. Reichman, L. B. Fletcher, N. W. Troy, and D. M. Krol: Opt. Mat. Express, 3, (2013) 502.

[30]H. Sugiura, and T. Yamadaya: J. Non Cryst. Solids, 144, (1992) 151.

[31] Y. Bellouard, E. Barthel, A. A. Said, M. Dugan, and P. Bado: Opt. Express, 16, (2008) 19520.

(Received: October 11, 2017, Accepted: January 20, 2018) 